\title{
Growth standards for nine North American fish species
}

\author{
Z. J. JACKSON \& M. C. QUIST \\ Department of Natural Resource Ecology and Management, Iowa State University, Ames, IA, USA
}

\author{
J. G. LARSCHEID \\ Iowa Department of Natural Resources, Spirit Lake, IA, USA
}

\begin{abstract}
Standard methods for comparing population characteristics within and among fish populations greatly enhance communications among fisheries scientists, improve the efficiency of data analysis, and provide insight that helps guide management actions. Although standard methods are available for comparing some fish population characteristics (e.g. length structure, body condition), similar methods are lacking for comparing growth. The purpose of this study was to provide standards (i.e. percentiles and a standard growth model) for nine ecologically and recreationally important fish species. Percentile distributions of mean back-calculated length at age were estimated using data obtained from the published literature and from data solicited from state and federal agencies throughout North America. Percentiles of growth were estimated for bluegill, Lepomis macrochirus Rafinesque, common carp, Cyprinus carpio Linnaeus, flathead catfish, Pylodictis olivaris (Rafinesque), largemouth bass, Micropterus salmoides (Lacepède), sauger, Sander canadensis (Griffith \& Smith), smallmouth bass, Micropterus dolomieu Lacepède, white bass, Morone chrysops (Rafinesque) and yellow perch, Perca flavescens (Mitchill). Standard growth models (i.e. von Bertalanffy models) were developed for these species and for channel catfish, Ictalurus punctatus (Rafinesque). These results provide tools that will help scientists compare growth of fishes across North America.
\end{abstract}

KEYWORDS: growth standards, relative growth index, von Bertalanffy.

\section{Introduction}

Evaluation of fish population characteristics in a system often includes comparison to regional or statewide averages or to some standard, and a variety of techniques have been developed that allow comparison. For example, proportional stock density (PSD) enables standardised assessment of the length structure of fish populations (Willis, Murphy \& Guy 1993), and relative weight $(\mathrm{Wr})$ provides a standardised assessment of fish condition among populations (Blackwell, Brown \& Willis 2000). Extensive comparisons of growth began with Carlander (1969, 1977, 1997), where mean back-calculated length at age and mean lengths at age were calculated by state and region. Hubert (1999) used a similar technique by calculating percentile values of mean lengths at age for 102 channel catfish, Ictalurus punctatus (Rafinesque), populations throughout North America for use as growth standards. Analogous methods were used to develop percentile standards for walleye, Sander vitreus (Mitchill) (Quist, Guy, Schultz \& Stephen 2003), white crappie, Pomoxis annularis Rafinesque, and black crappie, P. nigromaculatus (Lesueur) (Jackson \& Hurley 2005). Percentiles illustrate the range of observed lengths and describe the percentage of lengths smaller than the standard. Another method of interpreting growth information was described by Casselman \& Crossman (1986), who used von Bertalanffy growth models to estimate age-specific standard lengths for North American muskellunge, Esox masquinongy Mitchill, populations. This method was used to provide growth standards for walleye (Quist et al. 2003), black crappie and white crappie (Jackson \& Hurley 2005). 
Standard lengths $\left(L_{\mathrm{s}}\right)$ represent predicted age-specific lengths from a standard growth model (i.e. the von Bertalanffy model). Following Quist et al. (2003), agespecific standard lengths can be applied to the relative growth index [RGI $=\left(L_{t} / L_{\mathrm{s}}\right) \times 100$, where $L_{t}=$ observed length at age $(t)$ and $L_{\mathrm{s}}=$ predicted age-specific standard length]. This index can be used to summarise and interpret growth, with RGI values above 100 indicating fast growth and values below 100 indicating slower growth. Comparing growth to national averages or percentiles illustrates whether fish are growing faster or slower than other populations, as well as the relative magnitude of differences in growth. These evaluations can be used to provide insight on differences in growth based on environmental conditions (e.g. temperature, water quality, watershed protection) or management activities (e.g. harvest regulations, habitat improvements, trophic manipulations).

The full utility of growth standards is limited because they have only been developed for a few species. Given the potential utility of growth comparisons, the objectives of this study were to estimate percentile values of mean back-calculated length at age for North American populations of bluegill, Lepomis macrochirus Rafinesque, common carp, Cyprinus carpio Linnaeus, flathead catfish, Pylodictis olivaris (Rafinesque), largemouth bass, Micropterus salmoides (Lacepède), sauger, Sander canadensis (Griffith \& Smith), smallmouth bass, Micropterus dolomieu Lacepède, white bass, Morone chrysops (Rafinesque), and yellow perch, Perca flavescens (Mitchill). In addition, standard growth models, needed to calculate RGIs, were developed for each species. Only percentile values of growth had been calculated for channel catfish (Hubert 1999); therefore, a growth model was also developed for channel catfish. These species were selected because of their importance as sport fish (e.g. bluegill, largemouth bass) or their importance in affecting habitat quality (i.e. common carp; Crivelli 1983; Lougheed, Crosbie \& Chow-Fraser 1998; Miller $\&$ Crowl 2006). The common carp is native to Europe and Asia, but has been widely introduced throughout much of North America (Panek 1987). Bluegill, channel catfish, flathead catfish, largemouth bass, sauger, smallmouth bass, white bass and yellow perch are native to North America, but have also been introduced to many areas where they are not native.

\section{Methods}

Mean back-calculated length at age data for bluegill, channel catfish, common carp, flathead catfish, largemouth bass, sauger, smallmouth bass, white bass and yellow perch were assembled from the peer-reviewed literature. Additional information was obtained by soliciting state and federal natural resource agencies throughout North America. Growth data were based on a variety of structures including opercles, otoliths, pectoral spines, dorsal spines, scales and vertebrae. Although some structures may provide biased age estimates for older fish (Marwitz \& Hubert 1995; Kocovsky \& Carline 2000; Isermann, Meerbeek, Scholten \& Willis 2003), it was assumed the studies used structures that provided reasonably accurate estimates of age for the species and region. Growth data were only included from studies that contained at least 30 individuals. When multiple studies on a population were available, the study with the largest sample size was selected. As recognised by Hubert (1999), growth rates can vary substantially across the distribution of a species. Accordingly, it is important not only to include growth information from the entire distribution of a species, but also to minimise the influence of any one geographic region. Therefore, studies were limited to 10 populations from any state or province. Data were truncated for each species at the age when $25 \%$ of the populations did not include that age to minimise potential aging errors. For instance, if there were 100 populations of a given species, the oldest age present in at least 75 of the populations was the upper age limit used in the analysis. Further truncation occurred when growth standards were unreasonable (e.g. fifth percentile for age-7 bluegill was less than fifth percentile for age-6 bluegill).

Standards were computed from the distributions of mean back-calculated lengths at age for each species following Hubert (1999). Estimated percentiles of mean back-calculated lengths at age included the 5th, 10th, 25th, 50th, 75th, 90th and 95th percentiles. A von Bertalanffy growth model was estimated for each species using non-linear regression techniques (PROC NLIN) in Statistical Analysis System (SAS Institute 1996) following the method described in Quist et al. (2003). While sex-specific standards would be useful, available data rarely included information by sex ( $<5 \%$ of the populations). Thus, data were insufficient for calculating sex-specific standards.

\section{Results and discussion}

Growth data were obtained for 41 states and five Canadian provinces (Table 1). The number of populations from individual states or provinces varied from 1 to 60 (i.e. for all species), with South Dakota, Iowa, Oklahoma, Nebraska and Washington contributing the greatest number of populations. While the number 
Table 1. Number of populations of bluegill (BLG), common carp (CRP), flathead catfish (FHC), largemouth bass (LMB), sauger (SAU), smallmouth bass (SMB), white bass (WHB), and yellow perch (YEP) used in development of growth standards from each state and province

\begin{tabular}{|c|c|c|c|c|c|c|c|c|c|}
\hline State or province & BLG & CRP & FHC & LMB & SAU & SMB & WHB & YEP & Sources \\
\hline Alabama & & & 2 & 4 & & & & & $\begin{array}{l}\text { FHC: Nash (1999), M. Maceina, unpublished data; LMB: } \\
\text { Carlander (1977), D. Armstrong, unpublished data }\end{array}$ \\
\hline Arkansas & 6 & & & 4 & & 1 & 3 & & $\begin{array}{l}\text { BLG: Carlander (1977), S. Sammons, unpublished data; LMB: } \\
\text { Carlander (1977), Jackson et al. (2005); SMB: Carlander (1997); } \\
\text { WHB: Houser \& Bryant (1970), Yellayi \& Kilambi (1975), } \\
\text { Carlander (1997) }\end{array}$ \\
\hline Arizona & & & 3 & & & & & & FHC: Young \& Marsh (1990) \\
\hline California & 2 & & & 8 & & & & & BLG: Carlander (1977); LMB: Carlander (1977) \\
\hline Colorado & & & & & & 1 & & 1 & SMB: Patton \& Hubert (1996); YEP: Carlander (1997) \\
\hline Florida & & & & 10 & & & 1 & & LMB: Porak et al. (1986, 1987); WHB: Nordhaus et al. (1998) \\
\hline Georgia & 8 & & 3 & & & & & & $\begin{array}{l}\text { BLG: Carlander (1977), S. Sammons, unpublished data; } \\
\text { FHC: Quinn (1988), Nash (1999), Grabowski et al. (2004) }\end{array}$ \\
\hline Great Lakes & & & & & 1 & 5 & 1 & 4 & $\begin{array}{l}\text { SAU: Carlander (1997); SMB: Carlander (1977), } \\
\text { Danehy \& Ringler (1991); WHB: Carlander (1997); } \\
\text { YEP: Carlander (1997) }\end{array}$ \\
\hline Iowa & 10 & 10 & 2 & 10 & 3 & 7 & 7 & 6 & $\begin{array}{l}\text { BLG: Carlander (1977); CRP: Carlander (1969), Z. Jackson, } \\
\text { unpublished data; FHC: Mayhew (1969), Carlander (1977); } \\
\text { LMB: Carlander (1977), M. Steuck, unpublished data; } \\
\text { SAU: Carlander (1997), M. Steuck, unpublished data; } \\
\text { SMB: Carlander (1977), A. Jansen, unpublished data; } \\
\text { WHB: Sigler (1949), Pelren (1970), Carlander (1997), } \\
\text { M. Steuck, unpublished data; YEP: Carlander (1997) }\end{array}$ \\
\hline Idaho & 1 & & & 2 & & 3 & & & $\begin{array}{l}\text { BLG: Schriever et al. (1996); LMB: Schriever et al. (1996); } \\
\text { SMB: Carlander (1977) }\end{array}$ \\
\hline Illinois & 7 & & & 10 & 1 & 4 & 1 & & $\begin{array}{l}\text { BLG: Carlander (1977); LMB: Carlander (1977), } \\
\text { Storck et al. (1982), Perry \& Tranquilli (1984), } \\
\text { Garthause \& Heidinger (1999), } \\
\text { M. Steuck, unpublished data; SAU: Carlander (1997); } \\
\text { SMB: Carlander (1977); WHB: Carlander (1997) }\end{array}$ \\
\hline Indiana & 10 & & & 10 & & 2 & 1 & 3 & $\begin{array}{l}\text { BLG: Carlander (1997), Benson (2004), Carnahan (2005), } \\
\text { Doll (2005a,b), Robertson (2005), } \\
\text { M. Burlingame, unpublished data; } \\
\text { LMB: Benson (2004), Carnahan (2005), } \\
\text { Doll (2005a), Pearson (2005), Robertson (2005), } \\
\text { M. Burlingame, unpublished data, C. } \\
\text { Kowalik, unpublished data; SMB: Keller (2001), } \\
\text { Price \& Robertson (2005); WHB: Colvin (1993); } \\
\text { YEP: Benson (2004), Robertson (2005), } \\
\text { J. Doll, unpublished data }\end{array}$ \\
\hline Kansas & 4 & 2 & 4 & 3 & & & 10 & & $\begin{array}{l}\text { BLG: Cross et al. (1959), Delp et al. (2000); } \\
\text { CRP: Stucky \& Klaassen (1971); } \\
\text { FHC: Carlander (1977), Layher \& Boles (1979), } \\
\text { C. Paukert, unpublished data; LMB: Cross et al. (1959), } \\
\text { Delp et al. (2000); WHB: R. Schultz, unpublished data }\end{array}$ \\
\hline Kentucky & & & & 3 & & & 1 & & $\begin{array}{l}\text { LMB: Carlander (1977), Buynak et al. (1991), } \\
\text { Buynak \& Mitchell (1999); WHB: Carlander (1997) }\end{array}$ \\
\hline Louisiana & & & & 2 & & & & & LMB: Carlander (1977), Meador \& Kelso (1990) \\
\hline Massachusetts & & & & 1 & & 1 & & 6 & $\begin{array}{l}\text { LMB: Carlander (1977); SMB: Carlander (1977); } \\
\text { YEP: Carlander (1997) }\end{array}$ \\
\hline Manitoba & & & & & & & & 1 & YEP: Carlander (1997) \\
\hline Maryland & 1 & & & 1 & & 1 & & 1 & $\begin{array}{l}\text { BLG: Carlander (1977); LMB: Carlander (1977); SMB: } \\
\text { Carlander (1977); YEP: Carlander (1997) }\end{array}$ \\
\hline Maine & & & & & & 1 & & & SMB: Carlander (1977) \\
\hline Michigan & 5 & & & 1 & & & & & $\begin{array}{l}\text { BLG: Carlander (1977), Price (1977), Schneider (1997); } \\
\text { LMB: Carlander (1977) }\end{array}$ \\
\hline
\end{tabular}


Table 1. (Continued)

\begin{tabular}{|c|c|c|c|c|c|c|c|c|c|}
\hline State or province & BLG & CRP & FHC & LMB & SAU & SMB & WHB & YEP & Sources \\
\hline Minnesota & 8 & 1 & & 5 & 3 & 3 & & 4 & $\begin{array}{l}\text { BLG: Carlander (1977), Shroyer et al. (2003), J. } \\
\text { Meerbeek, unpublished data; CRP: Carlander (1969); } \\
\text { LMB: Carlander (1977), Smagula \& Adelman (1983), } \\
\text { Shroyer et al. (2003), J. Meerbeek, unpublished data; } \\
\text { SAU: Carlander (1997), J. Meerbeek, unpublished data; } \\
\text { SMB: Johnson \& Hale (1977), J. Meerbeek, unpublished data; } \\
\text { YEP: Carlander (1997) }\end{array}$ \\
\hline Missouri & 4 & 4 & 2 & 8 & & 2 & 1 & & $\begin{array}{l}\text { BLG: Serns \& Strawn (1975), Carlander (1977); } \\
\text { CRP: Purkett (1958), Carlander (1969); FHC: Purkett (1958), } \\
\text { K. Sullivan, unpublished data; LMB: Carlander (1977), } \\
\text { Novinger (1987); SMB: Carlander (1977); } \\
\text { WHB: Carlander (1997) }\end{array}$ \\
\hline Mississippi & & & 4 & 1 & & & & & $\begin{array}{l}\text { FHC: Insaurralde (1992), Francis (1993), } \\
\text { Schramm \& Eggleton (2006); LMB: } \\
\text { Miranda et al. (1987) }\end{array}$ \\
\hline Montana & 1 & & & 1 & 1 & & & 4 & $\begin{array}{l}\text { BLG: Brown \& Logan (1960); LMB: Brown \& Logan (1960); } \\
\text { SAU: Carlander (1997); YEP: Brown \& Logan (1960), } \\
\text { Carlander (1997) }\end{array}$ \\
\hline New Brunswick & & & & & & 1 & & & SMB: Carlander (1977) \\
\hline North Carolina & 10 & & 4 & 7 & & & & & $\begin{array}{l}\text { BLG: Carlander (1977); FHC: Guier et al. (1981), } \\
\text { Kwak et al. (2006); LMB: Carlander (1977) }\end{array}$ \\
\hline North Dakota & 1 & 2 & & 1 & 3 & 2 & 2 & 10 & $\begin{array}{l}\text { BLG: Kreft \& Power (1996); CRP: Nelson (1974), } \\
\text { Kreft \& Power (1996); LMB: Kreft \& Power (1996); } \\
\text { SAU: Carlander (1997); SMB: Kreft \& Power (1996); } \\
\text { WHB: Nelson (1974), R. Hiltner, unpublished data; } \\
\text { YEP: Kreft \& Power (1996), Carlander (1997) }\end{array}$ \\
\hline Nebraska & 10 & 1 & 2 & 10 & 3 & 3 & 10 & 10 & $\begin{array}{l}\text { BLG: K. Hurley, unpublished data; CRP: Hesse et al. (1978); } \\
\text { FHC: Hesse } \text { et al. (1978), Guier et al. (1981); } \\
\text { LMB: K. Hurley, unpublished data; SAU: } \\
\text { K. Hurley, unpublished data; SMB: K. Hurley, } \\
\text { unpublished data; WHB: Carlander (1997), } \\
\text { K. Hurley, unpublished data; YEP: Carlander (1997), } \\
\text { K. Hurley, unpublished data }\end{array}$ \\
\hline New Mexico & & & & 1 & & & 1 & & LMB: Carlander (1977); WHB: Carlander (1997) \\
\hline New York & & & & & & 2 & 1 & & SMB: Carlander (1977); WHB: Carlander (1997) \\
\hline Ohio & 1 & & & & 8 & 5 & & 2 & $\begin{array}{l}\text { BLG: Carlander (1977); SAU: S. Schell, unpublished data; } \\
\text { SMB: Carlander (1977); YEP: Carlander (1997) }\end{array}$ \\
\hline Oklahoma & 10 & 9 & 6 & 10 & & 9 & 10 & & $\begin{array}{l}\text { BLG: Carlander (1977); CRP: Houser (1956), } \\
\text { Jenkins \& Finnell (1957), Carlander (1969); } \\
\text { FHC: McCoy (1953), Jenkins \& Finnell (1957), } \\
\text { Carlander (1969), Turner (1982); LMB: Carlander (1977), } \\
\text { J. Long, unpublished data; SMB: Carlander (1977), } \\
\text { Orth et al. (1983), Gilliland et al. (1991), } \\
\text { J. Long, unpublished data; } \\
\text { WHB: Baglin \& Hill (1976), Carlander (1997) }\end{array}$ \\
\hline Ontario & & & & & & & & 1 & YEP: Sun \& Harvey (1986) \\
\hline Pennsylvania & 6 & & & 1 & & 3 & & 4 & $\begin{array}{l}\text { BLG: Carlander (1977); LMB: Carlander (1977); } \\
\text { SMB: Carlander (1977), Heisey et al. (1980); } \\
\text { YEP: Carlander (1997) }\end{array}$ \\
\hline Quebec & & & & & & & & 4 & YEP: Ridgway \& Chapleau (1994) \\
\hline South Carolina & 1 & & 2 & & & & & 1 & $\begin{array}{l}\text { BLG: Belk \& Hales (1993); } \\
\text { FHC: Bulak (1998); YEP: Carlander (1997) }\end{array}$ \\
\hline
\end{tabular}

of populations from each state or province was limited to 10 , it was rare that more than 10 populations per state or province were available (only 27 of 368 species- state or province combinations). The total number of populations varied from 35 to 147 among species, and all species except flathead catfish and sauger had more 
Table 1. (Continued)

\begin{tabular}{|c|c|c|c|c|c|c|c|c|c|}
\hline State or province & BLG & CRP & FHC & LMB & SAU & SMB & WHB & YEP & Sources \\
\hline South Dakota & 10 & 4 & 1 & 10 & 5 & 10 & 10 & 10 & $\begin{array}{l}\text { BLG: Carlander (1977), Willis et al. (2001); } \\
\text { CRP: Carlander (1969); FHC: G. Adams, } \\
\text { unpublished data; LMB: Carlander (1977), } \\
\text { Willis et al. (2001); SAU: Carlander (1997); } \\
\text { SMB: Willis et al. (2001), G. Adams, } \\
\text { unpublished data; WHB: Carlander (1997), } \\
\text { Soupir et al. (1997), Willis et al. (1997); YEP: Nelson (1974), } \\
\text { Willis et al. (2001), Brown \& St. Sauver (2002) }\end{array}$ \\
\hline Saskatchewan & & & & & & & & 1 & YEP: Carlander (1997) \\
\hline Tennessee & 3 & 1 & 1 & 6 & 6 & 4 & 4 & & $\begin{array}{l}\text { BLG: Carlander (1977); CRP: Carlander (1969); } \\
\text { FHC: Carroll \& Hall (1964); LMB: Carlander (1977); } \\
\text { SAU: Carlander (1997); SMB: Carlander (1977), } \\
\text { Marinac-Sanders \& Coble (1981); WHB: Myhr (1971), } \\
\text { Carlander (1997) }\end{array}$ \\
\hline Texas & 5 & & 1 & 4 & & 1 & 2 & & $\begin{array}{l}\text { BLG: Serns \& Strawn (1975), Carlander (1977); } \\
\text { FHC: Pate (1980); LMB: Carlander (1977); } \\
\text { SMB: Robertson \& Winemiller (2001); } \\
\text { WHB: Muoneke (1994), Carlander (1997) }\end{array}$ \\
\hline Utah & & 2 & & & & & 1 & 2 & $\begin{array}{l}\text { CRP: McConnell (1952), Carlander (1969); } \\
\text { WHB: Carlander (1997); YEP: Carlander (1997) }\end{array}$ \\
\hline Virginia & 1 & & & 1 & & 5 & 1 & 1 & $\begin{array}{l}\text { BLG: Smith \& Kauffman (1991); LMB: Carlander (1977); } \\
\text { SMB: Carlander (1977), Austen \& Orth (1988), } \\
\text { King et al. (1991); WHB: Colvin (1993); } \\
\text { YEP: Carlander (1997) }\end{array}$ \\
\hline Washington & 10 & & & 10 & & 10 & & 10 & $\begin{array}{l}\text { BLG: Mueller (1997a), Mueller \& Downen (1999a), } \\
\text { Downen \& Mueller (2000a,b), Mueller \& Downen (2000), } \\
\text { Jackson \& Caromile (2001), Osborne \& Petersen (2001), } \\
\text { Caromile \& Jackson (2002a), Couto \& Caromile (2006), } \\
\text { Petersen \& Osborne (2006); LMB: Mueller (1997a), } \\
\text { Mueller \& Downen (1999b), Downen \& Mueller (2000b), } \\
\text { Jackson \& Caromile (2000, 2001), Caromile \& Jackson (2002a), } \\
\text { Divens \& Osborne (2004, 2005), Couto \& Caromile (2006), } \\
\text { Petersen \& Osborne (2006); SMB: Mueller (1997b), } \\
\text { Downen \& Mueller (2000c,d), Mueller et al. (2001), } \\
\text { Caromile \& Jackson (2002b), Mueller et al. (2002), } \\
\text { Osborne, Divens and Baldwin (2003), } \\
\text { Osborne, Petersen and Jackson (2003), } \\
\text { Divens \& Osborne (2004), Woller et al. (2004); } \\
\text { YEP: Mueller (1997b), Downen et al. (1999), } \\
\text { Mueller \& Downen (1999b,c), Caromile et al. (2002), } \\
\text { Downen \& Mueller (2000b,d,e), Verhey \& Mueller (2001), } \\
\text { Verhey et al. (2001) }\end{array}$ \\
\hline Wisconsin & 10 & 4 & & 2 & 1 & 4 & 1 & 6 & $\begin{array}{l}\text { BLG: Parker (1958), Carlander (1977); CRP: Carlander (1969); } \\
\text { LMB: Parker (1958), Carlander (1977); SAU: Carlander (1997); } \\
\text { SMB: Carlander (1977), Marinac-Sanders \& Coble (1981); } \\
\text { WHB: Priegel (1971); YEP: Carlander (1997) }\end{array}$ \\
\hline West Virginia & & & 1 & & & 1 & & & FHC: Guier et al. (1981); SMB: Austen \& Orth (1988) \\
\hline Wyoming & & 1 & & & & 3 & & & CRP: Wichers (1976); SMB: Mullner \& Hubert (1993) \\
\hline Otoliths & 9 & 5 & 3 & 16 & & & & & \\
\hline Scales & 131 & & & 130 & 14 & 87 & 69 & 32 & \\
\hline Spines* & & 9 & 16 & & & & & & \\
\hline Other $^{\dagger}$ & 5 & 27 & 19 & 1 & 21 & 7 & & 60 & \\
\hline Total & 145 & 41 & 38 & 147 & 35 & 94 & 69 & 92 & \\
\hline
\end{tabular}

*Includes spines and fin rays.

Includes vertebrae, opercles, a combination of structures or data for which the structure was not reported. 
than 40 populations represented in the analysis. Flathead catfish and sauger have a limited spatial distribution in North America compared with the other species used in this study (Lee, Gilbert, Hocutt, Jenkins, McAllister \& Stauffer 1980), which likely led to the low number of populations available for this analysis. Largemouth bass and bluegill had the highest number of populations, probably because of their importance as sport fish and widespread distribution in North America (Lee et al. 1980). The resulting percentiles of mean back-calculated lengths at age are presented in Table 2 and growth models are provided in Table 3.

Age estimates for the mean back-calculated length at age data used in this study were completed using a

Table 2. Age, sample size (n), and percentile values of mean backcalculated lengths $(\mathrm{mm})$ for North American populations of eight fish species

\begin{tabular}{|c|c|c|c|c|c|c|c|c|}
\hline \multirow[b]{2}{*}{ Age } & \multirow[b]{2}{*}{$n$} & \multicolumn{7}{|c|}{ Percentiles } \\
\hline & & $5 \%$ & $10 \%$ & $25 \%$ & $50 \%$ & $75 \%$ & $90 \%$ & $95 \%$ \\
\hline \multicolumn{9}{|c|}{ Bluegill } \\
\hline 1 & 145 & 29 & 34 & 39 & 49 & 64 & 77 & 81 \\
\hline 2 & 145 & 58 & 61 & 74 & 94 & 111 & 124 & 136 \\
\hline 3 & 142 & 88 & 91 & 105 & 130 & 146 & 165 & 179 \\
\hline 4 & 137 & 112 & 119 & 135 & 152 & 173 & 193 & 203 \\
\hline 5 & 112 & 127 & 135 & 151 & 173 & 191 & 208 & 223 \\
\hline 6 & 70 & 137 & 145 & 163 & 185 & 206 & 221 & 233 \\
\hline \multicolumn{9}{|c|}{ Common carp } \\
\hline 1 & 41 & 87 & 95 & 120 & 132 & 173 & 236 & 290 \\
\hline 2 & 41 & 175 & 196 & 231 & 264 & 338 & 376 & 429 \\
\hline 3 & 39 & 226 & 249 & 306 & 348 & 437 & 505 & 518 \\
\hline 4 & 39 & 279 & 299 & 356 & 429 & 495 & 549 & 635 \\
\hline 5 & 39 & 338 & 338 & 402 & 488 & 556 & 577 & 691 \\
\hline 6 & 33 & 368 & 381 & 431 & 512 & 605 & 632 & 648 \\
\hline 7 & 32 & 401 & 419 & 451 & 556 & 639 & 658 & 696 \\
\hline 8 & 26 & 409 & 449 & 470 & 570 & 655 & 721 & 724 \\
\hline \multicolumn{9}{|c|}{ Flathead catfish } \\
\hline 1 & 38 & 61 & 76 & 89 & 132 & 164 & 230 & 260 \\
\hline 2 & 38 & 124 & 155 & 181 & 235 & 268 & 348 & 352 \\
\hline 3 & 38 & 211 & 231 & 251 & 324 & 382 & 479 & 509 \\
\hline 4 & 38 & 254 & 296 & 341 & 446 & 498 & 571 & 605 \\
\hline 5 & 38 & 280 & 340 & 437 & 554 & 621 & 678 & 710 \\
\hline 6 & 34 & 366 & 390 & 520 & 613 & 710 & 752 & 765 \\
\hline 7 & 33 & 409 & 442 & 603 & 676 & 773 & 821 & 833 \\
\hline 8 & 32 & 458 & 503 & 618 & 729 & 827 & 896 & 925 \\
\hline 9 & 25 & 500 & 511 & 675 & 762 & 902 & 1,000 & 1,003 \\
\hline \multicolumn{9}{|c|}{ Largemouth bass } \\
\hline 1 & 147 & 71 & 78 & 94 & 114 & 152 & 177 & 185 \\
\hline 2 & 147 & 134 & 153 & 179 & 216 & 264 & 287 & 312 \\
\hline 3 & 144 & 193 & 210 & 255 & 286 & 332 & 361 & 377 \\
\hline 4 & 137 & 238 & 264 & 310 & 340 & 388 & 419 & 445 \\
\hline 5 & 131 & 287 & 305 & 348 & 385 & 434 & 462 & 483 \\
\hline 6 & 110 & 332 & 345 & 385 & 426 & 474 & 506 & 523 \\
\hline 7 & 95 & 348 & 377 & 409 & 452 & 494 & 533 & 559 \\
\hline 8 & 73 & 376 & 403 & 442 & 472 & 511 & 559 & 582 \\
\hline
\end{tabular}

Table 2. (Continued)

\begin{tabular}{|c|c|c|c|c|c|c|c|c|}
\hline \multirow[b]{2}{*}{ Age } & \multirow[b]{2}{*}{$n$} & \multicolumn{7}{|c|}{ Percentiles } \\
\hline & & $5 \%$ & $10 \%$ & $25 \%$ & $50 \%$ & $75 \%$ & $90 \%$ & $95 \%$ \\
\hline \multicolumn{9}{|c|}{ Sauger } \\
\hline 1 & 35 & 122 & 126 & 143 & 188 & 229 & 241 & 252 \\
\hline 2 & 35 & 201 & 241 & 266 & 311 & 333 & 373 & 378 \\
\hline 3 & 30 & 266 & 295 & 341 & 371 & 396 & 428 & 442 \\
\hline 4 & 22 & 311 & 325 & 388 & 424 & 445 & 464 & 466 \\
\hline 5 & 17 & 315 & 347 & 406 & 461 & 485 & 514 & 517 \\
\hline \multicolumn{9}{|c|}{ Smallmouth bass } \\
\hline 1 & 94 & 68 & 74 & 82 & 90 & 99 & 111 & 118 \\
\hline 2 & 94 & 130 & 140 & 152 & 165 & 180 & 212 & 231 \\
\hline 3 & 93 & 167 & 184 & 214 & 236 & 259 & 297 & 332 \\
\hline 4 & 91 & 209 & 221 & 258 & 290 & 328 & 358 & 387 \\
\hline 5 & 78 & 239 & 263 & 297 & 337 & 379 & 420 & 437 \\
\hline 6 & 63 & 280 & 294 & 326 & 376 & 411 & 432 & 461 \\
\hline 7 & 46 & 281 & 310 & 356 & 398 & 427 & 457 & 473 \\
\hline \multicolumn{9}{|c|}{ White bass } \\
\hline 1 & 69 & 108 & 120 & 132 & 144 & 168 & 213 & 227 \\
\hline 2 & 67 & 204 & 210 & 226 & 257 & 286 & 340 & 349 \\
\hline 3 & 65 & 244 & 259 & 286 & 323 & 337 & 376 & 388 \\
\hline 4 & 62 & 272 & 288 & 322 & 356 & 375 & 416 & 421 \\
\hline 5 & 46 & 297 & 309 & 343 & 378 & 396 & 427 & 440 \\
\hline \multicolumn{9}{|c|}{ Yellow perch } \\
\hline 1 & 92 & 46 & 56 & 70 & 80 & 94 & 106 & 126 \\
\hline 2 & 92 & 94 & 104 & 114 & 134 & 163 & 177 & 186 \\
\hline 3 & 89 & 120 & 130 & 153 & 175 & 206 & 229 & 236 \\
\hline 4 & 86 & 144 & 153 & 180 & 204 & 235 & 251 & 264 \\
\hline 5 & 71 & 168 & 178 & 204 & 224 & 257 & 272 & 280 \\
\hline 6 & 52 & 175 & 200 & 225 & 245 & 264 & 282 & 310 \\
\hline
\end{tabular}

Table 3. Standard growth models for North American populations of nine fish species. For each model, age is fish age in years and $L_{\mathrm{s}}$ is the age-specific standard length in millimeters

\begin{tabular}{|c|c|c|c|}
\hline Species & Equation & $R^{2}$ & $P$-value \\
\hline Bluegill & $L_{\mathrm{s}}=225.0\left[1-\mathrm{e}^{-0.293(\text { age }-0.111)}\right]$ & 0.74 & $<0.0001$ \\
\hline $\begin{array}{c}\text { Channel } \\
\text { catfish }\end{array}$ & $L_{\mathrm{S}}=843.6\left[1-\mathrm{e}^{-0.096(\mathrm{age}+0.669)}\right]$ & 0.97 & $<0.0001$ \\
\hline $\begin{array}{l}\text { Common } \\
\text { carp }\end{array}$ & $L_{\mathrm{s}}=632.4\left[1-\mathrm{e}^{-0.283(\text { age }+0.053)}\right]$ & 0.65 & $<0.0001$ \\
\hline $\begin{array}{l}\text { Flathead } \\
\text { catfish }\end{array}$ & $L_{\mathrm{s}}=1,266.5\left[1-\mathrm{e}^{-0.103(\mathrm{age}+0.050)}\right]$ & 0.95 & $<0.0001$ \\
\hline $\begin{array}{l}\text { Largemouth } \\
\text { bass }\end{array}$ & $L_{\mathrm{s}}=550.0\left[1-\mathrm{e}^{-0.245(\mathrm{age}+0.053)}\right]$ & 0.97 & $<0.0001$ \\
\hline Sauger & $L_{\mathrm{s}}=477.8\left[1-\mathrm{e}^{-0.491(\text { age }+0.013)}\right]$ & 0.76 & $<0.0001$ \\
\hline $\begin{array}{l}\text { Smallmouth } \\
\text { bass }\end{array}$ & $L_{\mathrm{s}}=498.6\left[1-\mathrm{e}^{-0.229(\text { age }-0.141)}\right]$ & 0.97 & $<0.0001$ \\
\hline White bass & $L_{\mathrm{s}}=396.6\left[1-\mathrm{e}^{-0.565(\text { age }-0.113)}\right]$ & 0.78 & $<0.0001$ \\
\hline $\begin{array}{c}\text { Yellow } \\
\text { perch }\end{array}$ & $L_{\mathrm{s}}=280.5\left[1-\mathrm{e}^{-0.332(\text { age }+0.031)}\right]$ & 0.97 & $<0.0001$ \\
\hline
\end{tabular}

variety of hard structures. Certain structures (e.g. scales) have been shown to underestimate the age of fish, but the age at which this occurs varies among 
species (Marwitz \& Hubert 1995; Kocovsky \& Carline 2000; Isermann et al. 2003). These concerns were addressed by truncating the ages that were included in the analysis and compared percentiles of growth among structures to provide insight on potential biases associated with different structures. Although many have raised concerns that scales tend to overestimate growth by underestimating age (e.g. Isermann et al. 2003), results of the analysis indicated no major differences between structures used in this study. For example, the tenth percentile of age- 8 largemouth bass growth was $400 \mathrm{~mm}$ using scale data. The same percentile using otoliths was $404 \mathrm{~mm}$. The 50th percentile for age- 6 flathead catfish growth was $613 \mathrm{~mm}$ using otolith data and $617 \mathrm{~mm}$ using spines. Similar results were observed across all species and structures used in the analysis, suggesting that structures did not have a significant influence on the growth standards. Another possible concern regarding the data used in this study involves the use of different back-calculation techniques. While the use of several back-calculation techniques (i.e. Fraser-Lee, direct proportion) may increase variation in a data set, several studies have illustrated that errors associated with different back-calculation methods are minor. For example, Pierce, Rasmussen \& Leggett (1996) compared mean back-calculated lengths at age of pumpkinseed Lepomis gibbosus (Linneaus) and golden shiner Notemigonus crysoleucas (Mitchill) using three different back-calculation techniques (Fraser-Lee, one based on the scale proportion hypothesis, and one based on the body proportion hypothesis). Results of their study illustrated that growth estimates were nearly identical among techniques and similar to empirical observations of growth. Additionally, some researchers may prefer the use of mean length at age (i.e. at capture) to mean back-calculated length at age. However, such an approach has its own issues that may be more tenuous than those for back-calculated length at age. In particular, use of mean length at age would require that fish be sampled at the same time of year across North America. These data are unavailable and may cause more concern with regard to data structure and quality than back-calculated length at age.

The utility of RGI in evaluating differences in growth was illustrated by Quist et al. (2003) who concluded that percentiles provided a more descriptive interpretation of growth, while the RGI allowed for more refined analyses. As an example of how these standards can be used, bluegill populations in two natural lakes, West Okoboji Lake ( $n=60$ individuals) and Swan Lake, Iowa ( $n=52$ individuals; Z. Jackson, unpublished data) were examined. Mean backcalculated lengths of bluegills from West Okoboji were between the 25th and 50th percentiles at ages 1-3, indicating relatively slow growth at early ages. Growth increased at later ages where mean back-calculated lengths were between the 50th and 75th percentiles at age 4 , at the 75 th percentile at age 5 and between the 75 th and 90th percentiles at age 6 . In contrast, bluegills from Swan Lake were near the 25th percentile for all ages. The patterns are further illustrated by examining RGI values (Fig. 1). Bluegills exhibited slow growth for the first 3 years and then increased at older ages in West Okoboji Lake, whereas fish in Swan Lake were growing relatively slowly at all ages. Overall mean RGI values (i.e. across all ages) for each population were 96 in West Okoboji Lake (SD 16.0) and 86 in Swan Lake (SD 1.9). Differences in lake characteristics likely influenced differences in growth. West Okoboji Lake (1565 ha) is characterised as having good water quality (e.g. relatively low nutrients, high water clarity), whereas Swan Lake (57 ha) is characterised as having poor water quality (i.e. excessively high nutrient concentrations, low water clarity; J. Larscheid, unpublished data).

Comparing growth between lakes using standardised measures can aid in the identification of factors contributing to poor growth and help guide management actions. Jackson \& Hurley (2005) stated that RGI will likely take a similar path as other indices and become commonly used in the evaluation and communication of fish growth. However, this contention is based on the premise that standards are developed and available to scientists. The standards provided in this

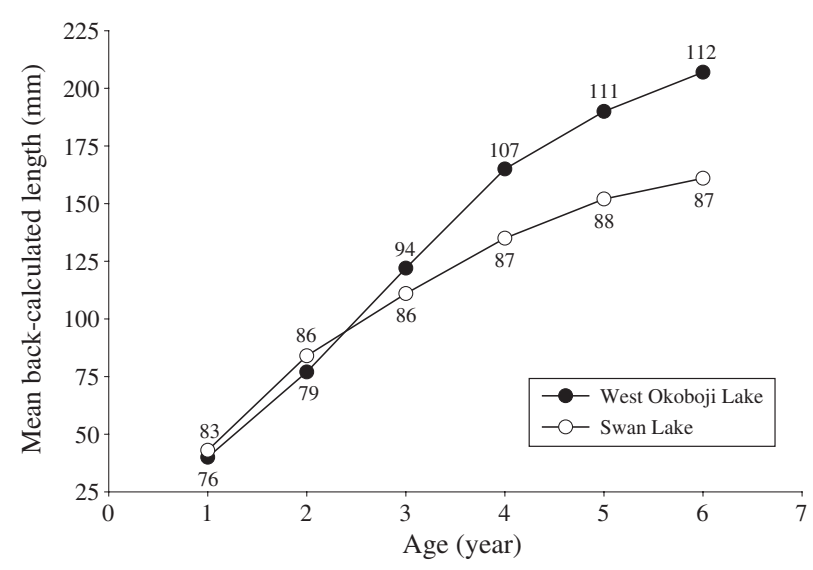

Figure 1. Mean back-calculated lengths and relative growth index (RGI; numbers near symbols) values at each age for bluegills sampled from two lakes in Iowa. 
study offer fisheries scientists another tool for identifying and establishing management goals, evaluating management objectives and providing insight into environmental conditions and management activities that affect growth. Revising and updating these standards may be required in the future as more data become available, but disseminating the standards to facilitate their application and evaluation by fisheries scientists is of foremost importance.

\section{Acknowledgments}

This project could not have been completed without data provided by G. Adams, D. Armstrong, J. Bailey, J. Breck, M. Burlingame, D. Dieterman, J. Doll, M. Drobish, M. Eggleton, R. Hiltner, K. Hurley, J. Jackson, A. Jansen, C. Kowalik, L. Kring, M. Kulp, J. Long, M. Maceina, J. MacMillan, J. Meerbeek, C. Paukert, J. Quinn, S. Sammons, S. Schell, H. Schramm, R. Schultz, M. Steuck, A. Stevens, K. Sullivan, and G. Thiede. We also thank S. Bonar, M. Brouder, W. Hubert, A. Iles, D. Simpkins, and D. Willis for useful discussions associated with growth standards. J. Koch, D. Rowe, and three anonymous reviewers provided helpful comments on a previous version of the manuscript. Funding was provided by Iowa Department of Natural Resources and the Department of Natural Resource Ecology and Management at Iowa State University.

\section{References}

Austen D.J. \& Orth D.J. (1988) Evaluation of a 305-mm minimum-length limit for smallmouth bass in the New River, Virginia and West Virginia. North American Journal of Fisheries Management 8, 231-239.

Baglin R.E. Jr \& Hill L.G. (1976) Age and growth characteristics of white bass Morone chrysops (Rafinesque), in Lake Texoma, Oklahoma. Texas Journal of Science 27, 113-124.

Belk M.C. \& Hales L.S. Jr. (1993) Predation-induced differences in growth and reproduction of bluegills (Lepomis macrochirus). Copeia 1993, 1034-1044.

Benson A.C. (2004) A Fisheries Survey of Shriner Lake, Whitley County, Indiana, June 2004. Indianapolis, IN: Indiana Department of Natural Resources, $18 \mathrm{pp}$.

Blackwell B.G., Brown M.L. \& Willis D.W. (2000) Relative weight $(\mathrm{Wr})$ status and current use in fisheries assessment and management. Reviews in Fisheries Science 8, 1-44.

Brown C.J.D. \& Logan S.M. (1960) Age and growth of four species of warm-water game fish from three Montana ponds. Transactions of the American Fisheries Society 89, 379-382.
Brown M.L. \& St. Sauver T. (2002) An assessment of yellow perch, Perca flavescens, stocking contributions in eastern South Dakota lakes. Fisheries Management and Ecology 9, 225-234.

Bulak J.S. (1998) Fisheries Investigations in Lakes and Streams - Statewide. Columbia, SC: South Carolina Department of Natural Resources, 16 pp.

Buynak G.L. \& Mitchell B. (1999) Contribution of stocked advanced-fingerling largemouth bass to the population and fishery at Taylorsville Lake, Kentucky. North American Journal of Fisheries Management 19, 494-503.

Buynak G.L., McLemore W.N. \& Mitchell B. (1991) Changes in largemouth bass populations at Kentucky and Barkley Lakes: environmental or regulatory responses? North American Journal of Fisheries Management 11, 285292.

Carlander K.D. (1969) Handbook of Freshwater Fishery Biology, Vol. 1. Ames, IA: Iowa State University Press, $752 \mathrm{pp}$.

Carlander K.D. (1977) Handbook of Freshwater Fishery Bio$\log y$, Vol. 2. Ames, IA: Iowa State University Press, 431 pp.

Carlander K.D. (1997) Handbook of Freshwater Fishery Biology, Vol. 3. Ames, IA: Iowa State University Press, 397 pp.

Carnahan D.P. (2005) Patoka Lake, 2004 Fish Management Report. Indianapolis, IN: Indiana Department of Natural Resources, 48 pp.

Caromile S.J. \& Jackson C.S. (2002a) 2000 Warmwater Fish Survey of Silver Lake, Cowlitz County. Olympia, WA: Washington Department of Fish and Wildlife, $37 \mathrm{pp}$.

Caromile S.J. \& Jackson C.S. (2002b) Warmwater Fish Survey of Spanaway Lake, Pierce County, Spring 2000. Olympia, WA: Washington Department of Fish and Wildlife, 29 pp.

Caromile S.J., Jackson C.S. \& Meyer W. (2002) 1999 Warmwater Fish Survey of Harts Lake, Pierce County. Olympia, WA: Washington Department of Fish and Wildlife, 34 pp.

Carroll B.B. \& Hall G.E. (1964) Growth of catfishes in Norris Reservoir, Tennessee. Journal of the Tennessee Academy of Science 39, 86-91.

Casselman J.M. \& Crossman E.J. (1986) Size, age, and growth of trophy muskellunge and muskellunge-northern pike hybrids - the Cleithrum Project 1979-1983. In: G.E. Hall (ed.) Managing Muskies. Bethesda, MD: American Fisheries Society, pp. 93-110.

Colvin M.A (1993) Ecology and Management of White Bass: A Literature Review. Columbia, MO: Missouri Department of Conservation, $62 \mathrm{pp}$.

Couto A. \& Caromile S. (2006) The Warmwater Fish Community of Duck Lake, Grays Harbor County 1999-2004. Olympia, WA: Washington Department of Fish and Wildlife, $41 \mathrm{pp}$. 
Crivelli V.J. (1983) The destruction of aquatic vegetation by carp. Hydrobiologia 106, 37-41.

Cross F.B., Deacon J.E. \& Ward C.M. (1959) Growth data on sport fishes in twelve lakes in Kansas. Transactions of the Kansas Academy of Science 62, 162-164.

Danehy R.J. \& Ringler N.H. (1991) Ecology of smallmouth bass (Micropterus dolomieui) in the nearshore of Mexico Bay, Lake Ontario. In: D.C. Jackson (ed.) The First International Smallmouth Bass Symposium. Starkville, MS: Mississippi State University, pp. 66-72.

Delp J.G., Tillma J.S., Quist M.C. \& Guy C.S. (2000) Age and growth of four centrarchid species in southeastern Kansas streams. Journal of Freshwater Ecology 15, 475478.

Divens M. \& Osborne R. (2004) 2002 Warmwater Fisheries Survey of Chapman Lake (Spokane County). Olympia, WA: Washington Department of Fish and Wildlife, $30 \mathrm{pp}$.

Divens M. \& Osborne R. (2005) 2003 Warmwater Fisheries Surveys of Upper and Lower Twin Lakes (Lincoln County). Olympia, WA: Washington Department of Fish and Wildlife, $36 \mathrm{pp}$.

Doll J.C. (2005a) Deer Creek Lake, 2004 Fish Management Report. Indianapolis, IN: Indiana Department of Natural Resources, $13 \mathrm{pp}$.

Doll J.C. (2005b) Scott Township Park Pond, 2004 Fish Management Report. Indianapolis, IN: Indiana Department of Natural Resources, 12 pp.

Downen M.R. \& Mueller K.W. (2000a) 1999 Lake Goodwin Survey: Potential Trophy Largemouth Bass and Smallmouth Bass Fisheries in a Heavily Fished, Intensively Managed Western Washington lake. Olympia, WA: Washington Department of Fish and Wildlife, $39 \mathrm{pp}$.

Downen M.R. \& Mueller K.W. (2000b) 1998 Lake Hummel Survey: the Largemouth Bass-bluegill Community of a Eutrophic Island Lake. Olympia, WA: Washington Department of Fish and Wildlife, 30 pp.

Downen M.R. \& Mueller K.W. (2000c) 1999 Lake Terrell Survey: Potential Trophy Largemouth Bass and Channel Catfish Fisheries in a Lowland Western Washington Lake Preserve. Olympia, WA: Washington Department of Fish and Wildlife, $36 \mathrm{pp}$.

Downen M.R. \& Mueller K.W. (2000d) 1999 Lake Sawyer Survey: the Warmwater Fish Community in a Popular, Unregulated Fishery. Olympia, WA: Washington Department of Fish and Wildlife, $39 \mathrm{pp}$.

Downen M.R. \& Mueller K.W. (2000e) 1999 Campbell Lake Survey: the Warmwater Fish Community Fifteen Years after Implementation of a Lake Restoration Plan. Olympia, WA: Washington Department of Fish and Wildlife, 37 pp.

Downen M.R., Mueller K.W. \& Fletcher D. (1999) 1998 Lake Cassidy Survey: a Warmwater Fish Community Competing under Conditions of Hypolimnetic Anoxia and
Dense Aquatic Macrophytes. Olympia, WA: Washington Department of Fish and Wildlife, 32 pp.

Francis J.M. (1993) Recreational Handgrabbing as a Factor Influencing Flathead Catfish Stock Characteristics in Two Mississippi Streams. MSc thesis, Starkville, MS: Mississippi State University, 90 pp.

Garthause M.W. \& Heidinger R.C. (1999) Response of largemouth bass and bluegill to length limits in Lake of Egypt. Transactions of the Illinois State Academy of Science 92, 79-87.

Gilliland E., Horton R., Hysmith B. \& Moczygemba J. (1991) Smallmouth bass in Lake Texoma, a case history. In: D.C. Jackson (ed.) The First International Smallmouth Bass Symposium. Starkville, MS: Mississippi State University, pp. 136-142.

Grabowski T.B., Isely J.J. \& Weller R.R. (2004) Age and growth of flathead catfish, Pylodictus olivaris Rafinesque, in the Altamaha River system, Georgia. Journal of Freshwater Ecology 19, 411-417.

Guier C.R., Nichols L.E. \& Rachels R.T. (1981) Biological investigation of flathead catfish in the Cape Fear River. Proceedings of the Annual Conference Southeastern Association of Fish and Wildife Agencies 35, 607-621.

Heisey P.G., Mathur D. \& Magnusson N.C. (1980) Accelerated growth of smallmouth bass in a pumped storage system. Transactions of the American Fisheries Society 109, 371-377.

Hesse L.W., Wallace C.R. \& Lehman L. (1978) Fishes of the Channelized Missouri: Age-growth, Length-frequency, Length-weight, Coefficient of Condition, Catch Curves and Mortality of 25 Species of Channelized Missouri River Fishes. Norfolk, NE: Nebraska Game and Parks Commission, $61 \mathrm{pp}$.

Houser A. (1956) Continued Age and Growth Studies of Principal Fishes in Tenkiller Reservoir. Norman, OK: Oklahoma Fishery Research Laboratory, $21 \mathrm{pp}$.

Houser A. \& Bryant H.E. (1970) Age, growth, sex composition, and maturity of white bass in Bull Shoals Reservoir. U.S. Fish and Wildlife Service Technical Paper 49, 11 pp.

Hubert W.A. (1999) Standards for assessment of age and growth data for channel catfish. Journal of Freshwater Ecology 14, 313-326.

Insaurralde M.S. (1992) Environmental Characteristics Associated with Flathead Catfish in Four Mississippi Streams. Doctoral dissertation, Starkville, MS: Mississippi State University, $152 \mathrm{pp}$.

Isermann D.A., Meerbeek J.R., Scholten G.D. \& Willis D.W. (2003) Evaluation of three different structures used for walleye age estimation with emphasis on removal and processing times. North American Journal of Fisheries Management 23, 625-631.

Jackson C.S. \& Caromile S.J. (2000) An Assessment of the Warmwater Fish Community in Leland Lake, September 
1999. Olympia, WA: Washington Department of Fish and Wildlife, 25 pp.

Jackson C.S. \& Caromile S.J. (2001) An Assessment of the Warmwater Fish Community in Lake St. Clair (Thurston County) June 2000, Olympia, WA: Washington Department of Fish and Wildlife, $24 \mathrm{pp}$.

Jackson J.J. \& Hurley K.L. (2005) Relative growth of white crappie and black crappie in the United States. Journal of Freshwater Ecology 20, 461-467.

Jackson J.R., Hungerford T. \& Sanders S. (2005) Effects of Increased Minimum Flow and Stream Temperature on the Fishes of the Ouachita River. Little Rock, AR: Arkansas Game and Fish Commission, 129 pp.

Jenkins R.M. \& Finnell J.C. (1957) The Fisheries Resources of the Verdigris River in Oklahoma. Norman, OK: Oklahoma Fishery Research Laboratory, $46 \mathrm{pp}$.

Johnson F.H. \& Hale J.G. (1977) Interactions between walleye (Stizostedion vitreum vitreum) and smallmouth bass (Micropterus dolomieui) in four northeastern Minnesota lakes, 1948-69. Journal of the Fisheries Research Board of Canada 34, 1626-1632.

Keller D.C. (2001) Evaluation of Game Fish Populations in a Portion of Fall Creek. Indianapolis, IN: Indiana Department of Natural Resources Fish and Wildlife, 7 pp.

King M.A., Graham R.J. \& Woolcott W.S. (1991) Comparison of smallmouth bass from two tributaries of the York River, Virginia. In: D.C. Jackson (ed.) The First International Smallmouth Bass Symposium. Starkville, MS: Mississippi State University, pp. 6-13.

Kocovsky P.M. \& Carline R.F. (2000) A comparison of methods for estimating ages of unexploited walleyes. North American Journal of Fisheries Management 20, 1044-1048.

Kreft B.L. \& Power G.J. (1996) Statewide Biological Parameters for North Dakota's Fisheries. Bismarck, ND: North Dakota Game and Fish Department, 42 pp.

Kwak T.J., Pine W.E. III \& Waters D.S. (2006) Age, growth, and mortality of introduced flathead catfish in Atlantic rivers and a review of other populations. North American Journal of Fisheries Management 26, 73-87.

Layher W.G. \& Boles R.J. (1979) Growth of Pylodictus olivaris (Rafinesque) in a Kansas Reservoir. Transactions of the Kansas Academy of Sciences 82, 36-48.

Lee D.S., Gilbert C.R., Hocutt C.H., Jenkins R.E., McAllister D.E. \& Stauffer J.R. Jr. (1980) Atlas of North American Freshwater Fishes. Raleigh, NC: North Carolina State Museum of Natural History, 867 pp.

Lougheed V.L., Crosbie B. \& Chow-Fraser P. (1998) Predictions on the effect of common carp (Cyprinus carpio) exclusion on water quality, zooplankton, and submergent macrophytes in a Great Lakes wetland. Canadian Journal of Fisheries and Aquatic Sciences 55, 1189-1197.

Marinac-Sanders P. \& Coble D.W. (1981) The smallmouth bass population and fishery in a northern Wisconsin lake, with implications for other waters. North American Journal of Fisheries Management 1, 15-20.

Marwitz T.D. \& Hubert W.A. (1995) Precision of age estimates of Wyoming walleyes from different calcified structures. Prairie Naturalist 27, 41-49.

Mayhew J.K. (1969) Age and growth of flathead catfish in the Des Moines River, Iowa. Transactions of the American Fisheries Society 98, 118-120.

McConnell W.J. (1952) The opercular bone as an indicator of age and growth of the carp, Cyprinus carpio Linnaeus. Transactions of the American Fisheries Society 81, 138-149.

McCoy H.A. (1953) The rate of growth of flathead catfish from twenty-one Oklahoma lakes. Proceedings of the Oklahoma Academy of Science 33, 47-52.

Meador M.R. \& Kelso W.E. (1990) Growth of largemouth bass in low-salinity environments. Transactions of the American Fisheries Society 119, 545-552.

Miller S.A. \& Crowl T.A. (2006) Effects of common carp (Cyprinus carpio) on macrophytes and invertebrate communities in a shallow lake. Freshwater Biology 51, 85-94.

Miranda L.E., Wingo W.M., Muncy R.J. \& Bates T.D. (1987) Bias in growth estimates derived from fish collected by anglers. In: R.C. Summerfelt (ed.) The Age and Growth of Fish. Ames, IA: Iowa State University Press, pp. 211220.

Mueller K.W. (1997a) 1997 Fazon Lake Survey: Crowding of the Warmwater Fish Community in a Small, Lowland Lake. Olympia, WA: Washington Department of Fish and Wildlife, 23 pp.

Mueller K.W. (1997b) Lake Stevens Survey: The Warmwater Fish Community after Implementation of a Minimum Length Limit on Largemouth and Smallmouth Bass. Olympia, WA: Washington Department of Fish and Wildlife, 26 pp.

Mueller K.W. \& Downen M.R. (1999a) 1997 Lacamas Lake Survey: The Warmwater Fish Community of a Highly Eutrophic Lowland Lake. Olympia, WA: Washington Department of Fish and Wildlife, 33 pp.

Mueller K.W. \& Downen M.R. (1999b) 1997 Big Lake Survey: The Warmwater Fish Community before Treatment with a Selective Herbicide. Olympia, WA: Washington Department of Fish and Wildlife, $32 \mathrm{pp}$.

Mueller K.W. \& Downen M.R. (1999c) 1997 American Lake Survey: The Warmwater Fish Community before Stocking Smallmouth Bass. Olympia, WA: Washington Department of Fish and Wildlife, $35 \mathrm{pp}$.

Mueller K.W. \& Downen M.R. (2000) 1997 Horseshoe Lake Survey: The Warmwater Fish Community before Chemical Precipitation of Phosphorus and Increased Dilution of the Lake. Olympia, WA: Washington Department of Fish and Wildlife, 39 pp.

Mueller K.W., Serdar D.M. \& McBride D.E. (2001) Mercury in Sportfishes of Lake Whatcom, Washington, Including a 
Review of Potential Impacts to Aquatic Resources and People. Olympia, WA: Washington Department of Fish and Wildlife, 59 pp.

Mueller K.W., Brouwer N.L. \& Congdon B.D. (2002) 2001 Spencer Lake Survey: Biological Characteristics of a Minimally Exploited, Isolated Fish Community Consisting of Smallmouth Bass, Largemouth Bass, and Prickly Sculpin. Olympia, WA: Washington Department of Fish and Wildlife, $53 \mathrm{pp}$.

Mullner S.A. \& Hubert W.A. (1993) Growth of smallmouth bass (Micropterus dolomieu) in Flaming Gorge Reservoir, Wyoming-Utah. Great Basin Naturalist 53, 180-185.

Muoneke M.I. (1994) Dynamics of a heavily exploited Texas white bass population. North American Journal of Fisheries Management 14, 415-422.

Myhr A.I. III (1971) A Study of the White Bass, Morone chrysops (Rafinesque), in Dale Hollow Reservoir, Tennessee-Kentucky. MSc thesis, Cookeville, TN: Tennessee Technological University, 58 pp.

Nash M.K. (1999) Age and Growth of Flathead Catfish and Channel Catfish in the Tallapoosa River, Alabama and the Ocmulgee River, Georgia. MSc thesis, Auburn, AL: Auburn University, 75 pp.

Nelson W.R. (1974) Age, growth, and maturity of thirteen species of fish from Lake Oahe during the early years of impoundment, 1963-68. U.S. Fish and Wildlife Service Technical Paper 77, $29 \mathrm{pp}$.

Nordhaus J.J., Lubinski B.R., Cailteux R.L. \& Dobbins D.A. (1998) Age and growth of a newly established white bass population in Florida. Florida Scientist 61, 188-194.

Novinger G.D. (1987) Evaluation of a 15.0-inch minimum length limit on largemouth bass and spotted bass catches at Table Rock Lake, Missouri. North American Journal of Fisheries Management 7, 260-272.

Orth D.J., Oakey D.D. \& Maughan O.E. (1983) Population characteristics of smallmouth bass in Glover Creek, Southeast Oklahoma. Proceedings of Oklahoma Academy of Science 63, 37-41.

Osborne R.S. \& Petersen M.R. (2001) 2000 Warmwater Fisheries Survey of Whitestone Lake, Okanogan County, Washington. Olympia, WA: Washington Department of Fish and Wildlife, $40 \mathrm{pp}$.

Osborne R.S., Divens M.J. \& Baldwin C. (2003) 2001 Warmwater Fisheries Survey of Lake Spokane, Spokane and Stevens Counties, Washington. Olympia, WA: Washington Department of Fish and Wildlife, 58 pp.

Osborne R.S., Petersen M.R. \& Jackson C.S. (2003) 1999 Warmwater Fisheries Survey of Palmer Lake (Okanogan County), Washington. Olympia, WA: Washington Department of Fish and Wildlife, 42 pp.

Panek F.M. (1987) Biology and ecology of carp. In: E.L. Cooper (ed.) Carp in North America. Bethesda, MD: American Fisheries Society, pp. 1-15.
Parker R.A. (1958) Some effects of thinning on a population of fishes. Ecology 39, 304-317.

Pate M.W. (1980) Age and growth of flathead catfish, $P y$ lodictis olivaris, in the Rio Grande River, Texas. Annual Proceedings of the Texas Chapter, American Fisheries Society 3, 92-102.

Patton T.M. \& Hubert W.A. (1996) Water temperature affects smallmouth bass and channel catfish in a tailwater stream on the Great Plains. North American Journal of Fisheries Management 16, 124-131.

Pearson J. (2005) Impacts of Predator Management on Bluegill Fishing at Loon Lake, Indiana. Indianapolis, IN: Indiana Department of Natural Resources, 29 pp.

Pelren D.W. (1970) Age and growth of white bass from Pool 19 of the Mississippi River. Iowa State Journal of Science 44, 471-479.

Perry L.G. \& Tranquilli J.A. (1984) Age and growth of largemouth bass in a thermally altered reservoir, as determined from otoliths. North American Journal of Fisheries Management 4, 321-330.

Petersen M.R. \& Osborne R.S. (2006) 2001 Washburn Island Pond Warmwater Survey Okanogan County, Washington. Olympia, WA: Washington Department of Fish and Wildlife, $39 \mathrm{pp}$.

Pierce C.L., Rasmussen J.B. \& Leggett W.C. (1996) Backcalculation of fish length from scales: empirical comparison of proportional methods. Transactions of the American Fisheries Society 125, 889-898.

Porak W., Coleman W.S. \& Crawford S. (1986) Age, growth, and mortality of Florida largemouth bass utilizing otoliths. Proceedings of the Annual Conference Southeastern Association of Fish and Wildlife Agencies 29, 172-184.

Porak W.F., Crawford S. \& Estes J.R. (1987) Largemouth Bass Investigations. Tallahassee, FL: Florida Game and Fresh Water Fish Commission, 98 pp.

Price R. (1977) Age and growth of an unbalanced bluegill population in Michigan. Transactions of the American Fisheries Society 106, 331-333.

Price J. \& Robertson B. (2005) Fishery, Habitat, and Recreational Use Surveys for the Kankakee River, Indiana. Indianapolis, IN: Indiana Department of Natural Resources, $31 \mathrm{pp}$.

Priegel G.R. (1971) Age and rate of growth of the white bass in Lake Winnebago, Wisconsin. Transactions of the American Fisheries Society 100, 567-569.

Purkett C.A. Jr. (1958) Growth of the fishes in the Salt River, Missouri. Transactions of the American Fisheries Society 87, 116-131.

Quinn S.P. (1988) Investigations into the Biology and Potential Fishery for the Flathead Catfish (Pylodictis olivaris) in the Lower Flint River. Atlanta, GA: Georgia Department of Natural Resources, $47 \mathrm{pp}$. 
Quist M.C., Guy C.S., Schultz R.D. \& Stephen J.L. (2003) Latitudinal comparison of walleye growth in North America and factors influencing growth of walleyes in Kansas reservoirs. North American Journal of Fisheries Management 23, 677-692.

Ridgway L.L. \& Chapleau F. (1994) Study of a stunted population of yellow perch (Perca flavescens) in a monospecific lake in Gatineau Park, Quebec. Canadian Journal of Zoology 72, 1576-1582.

Robertson B. (2005) Worster Lake Fish Population Survey. Indianapolis, IN: Indiana Department of Natural Resources, $22 \mathrm{pp}$.

Robertson M.S. \& Winemiller K.O. (2001) Diet and growth of smallmouth bass in the Devils River, Texas. The Southwestern Naturalist 46, 216-221.

SAS Institute (1996) SAS/STAT User's Guide for Personal Computers, Version 6. Cary, NC: SAS Institute 1. 686 pp.

Schneider J.C. (1997) The 1997 Fish Survey of Rush Lake. Ann Arbor, MI: Michigan Department of Natural Resources Fisheries Division, 20 pp.

Schramm H.L. Jr. \& Eggleton M.A. (2006) Applicability of the flood-pulse concept in a temperate floodplain river ecosystem: thermal and temporal components. River Research and Applications 22, 543-553.

Schriever E., Brostrom J. \& Cochnauer T.E. (1996) River and Stream Investigations. Boise, ID: Idaho Department of Fish and Game, 142 pp.

Serns S.L. \& Strawn K. (1975) Age and growth of bluegill, Lepomis macrochirus, in two heated Texas reservoirs. Transactions of the American Fisheries Society 104, 506-512.

Shroyer S.M., Bandow F.L. \& Logsdon D.E. (2003) Effects of Prohibiting Harvest of Largemouth Bass on the Largemouth Bass and Bluegill Fisheries in Two Minnesota lakes. St. Paul, MN: Minnesota Department of Natural Resources, $47 \mathrm{pp}$.

Sigler W.F. (1949) Life history of the white bass, Lepibema chrysops (Rafinesque), of Spirit Lake, Iowa. Iowa State College Journal of Science 23, 311-316.

Smagula C.M. \& Adelman I.R. (1983) Growth in a natural population of largemouth bass, Micropterus salmoides Lacepede, as determined by physical measurements and [14C]-glycerine uptake by scales. Journal of Fish Biology 22, 695-703.

Smith P.P. \& Kauffman J.W. (1991) The effects of a slot size limit regulation on smallmouth bass in the Shenandoah River, Virginia. In: D.C. Jackson (ed.) The First International Smallmouth Bass Symposium. Starkville, MS: Mississippi State University, pp. 112-117.

Soupir C.A., Blackwell B.B. \& Brown M.L. (1997) Relative precision among calcified structures for white bass age and growth assessment. Journal of Freshwater Ecology 12, 531538.
Storck T., Dimond B. \& Miller S. (1982) Determination of Factors Affecting the Survival of Larval Fish and an Evaluation of Their Utilization as Food by Predators. Springfield, IL: Illinois Department of Conservation, $66 \mathrm{pp}$.

Stucky N.P. \& Klaassen H.E. (1971) Growth and condition of the carp and the river carpsucker in an altered environment in western Kansas. Transactions of the American Fisheries Society 100, 276-282.

Sun J. \& Harvey H.H. (1986) Population dynamics of yellow perch (Perca flavescens) and pumpkinseed (Lepomis gibbosus) in two acid-stressed lakes. Water, Air, and Soil Pollution 30, 611-617.

Turner P.R. (1982) Procedures for age determination and growth rate calculations of flathead catfish. Proceedings of the Annual Conference Southeastern Association of Fish and Wildlife Agencies 34, 253-262.

Verhey P.A. \& Mueller K.W. (2001) 2000 Lake Meridian Survey: The Warmwater Fish Community of an Oligotrophic Urban Lake. Olympia, WA: Washington Department of Fish and Wildlife, 45 pp.

Verhey P.A., Mueller K.W. \& Downen M.R. (2001) The Warmwater Fish Community of Lake Roesiger, with Notes on Its Fisheries History. Olympia, WA: Washington Department of Fish and Wildlife, $51 \mathrm{pp}$.

Wichers W.F. (1976) Age and Growth of Carp (Cyprinus carpio), from Pathfinder Reservoir, Wyoming, 1974 and 1975. MSc thesis, Laramie, WY: University of Wyoming, $69 \mathrm{pp}$.

Willis D.W., Murphy B.R. \& Guy C.S. (1993) Stock density indices: development, use, and limitations. Reviews in Fisheries Science 1, 203-222.

Willis D.W., Beck H.D., Soupir C.A., Johnson B.A., Simpson G.D. \& Wickstrom G.A. (1997) White bass growth in South Dakota waters. Prairie Naturalist 29, 111118.

Willis D.W., Isermann D.A., Hubers M.J., Johnson B.A., Miller W.H., St. Sauver T.R., Sorensen J.S., Unkenholz E.G. \& Wickstrom G.A. (2001) Growth of South Dakota Fishes: A Statewide Summary with Means by Region and Water Type. Pierre, SD: South Dakota Department of Game, Fish and Parks Fisheries Division, 46 pp.

Woller H., Baldwin C., Polacek M., Knuttgen K., Caromile S. \& Jackson C. (2004) Banks Lake Fish Survey, September 2000. Olympia, WA: Washington Department of Fish and Wildlife, $71 \mathrm{pp}$.

Yellayi R.R. \& Kilambi R.V. (1975) Population dynamics of white bass in Beaver Reservoir, Arkansas. Proceedings of the Annual Conference Southeastern Association of Fish and Wildlife Agencies 29, 172-184.

Young K.L. \& Marsh P.C. (1990) Age and growth of flathead catfish in four southwestern rivers. California Fish and Game 76, 224-233. 